\title{
Optical generation of vortices in trapped Bose-Einstein condensates
}

\author{
Ł. Dobrek, ${ }^{1}$ M. Gajda, ${ }^{1,2}$ M. Lewenstein, ${ }^{3}$ K. Sengstock, ${ }^{4}$ G. Birkl,${ }^{4}$ and W. Ertmer ${ }^{4}$ \\ ${ }^{1}$ College of Sciences, Aleja Lotników 32, 02668 Warsaw, Poland \\ ${ }^{2}$ Institute of Physics, Polish Academy of Sciences, 02668 Warsaw, Poland \\ ${ }^{3}$ Institut für Theoretische Physik, Universität Hannover, 30167 Hannover, Germany \\ ${ }^{4}$ Institut für Quantenoptik, Universität Hannover, 30167 Hannover, Germany
}

(Received 7 July 1999)

\begin{abstract}
We demonstrate numerically the efficient generation of vortices in Bose-Einstein condensates (BECs) by using a "phase imprinting" method. The method consists of passing a far-off-resonant laser pulse through an absorption plate with an azimuthally dependent absorption coefficient, imaging the laser beam onto a BEC, and thus creating the corresponding nondissipative Stark-shift potential and condensate phase shift. In our calculations we take into account experimental imperfections. We also propose an interference method to detect vortices by coherently pushing part of the condensate using optically induced Bragg scattering.
\end{abstract}

[S1050-2947(99)50211-4]

PACS number(s): 03.75.Fi, 32.80.Pj, 42.50.Vk

One of the remaining challenges of the physics of trapped Bose-Einstein condensates (BECs) [1-3] concerns the demonstration of their superfluid behavior. Superfluidity is inevitably related to the existence of vortices and persistent currents in BEC, which so far have not been observed experimentally, despite serious efforts [4]. Two aspects of the vortex problem have been studied intensively. (i) In rotating traps vortices appear in a natural way as thermodynamic ground states with quantized angular momentum [5]. Stability and other properties of vortices in rotating traps have been throughly discussed in Refs. [6-8]. (ii) In stationary traps the creation of vortices (or related dark solitons in one-dimension) requires the use of dynamical means, and an independent stability analysis.

Several methods were proposed to generate vortices in nonrotating traps: stirring the condensate using a blue detuned laser $[9,10]$, or several laser beams [11]; adiabatic passage [12] or Raman transitions [13] in bicondensate systems. Such vortices are typically not stable, and can exhibit dynamical or energetic instability. In the first case vortices decay rapidly, in the second the vortices are stable within the framework of the mean field theory, and their corresponding decay requires that one take into account interactions between the BEC and the thermal cloud. In the latter case the vortex dynamics is expected to be sufficiently slow, and thus experimentally accessible [14].

In this Rapid Communication we propose and investigate yet another procedure of vortex generation using "phase imprinting." This method consists of (i) passing a far-offresonant laser pulse through an absorption plate whose absorption coefficient depends on the rotation angle $\varphi$ around the propagation axis and ii) creating the corresponding conservative Stark-shift potential that leads to a $\varphi$-dependent phase shift in the condensate wave function. This method is very efficient and robust, and allows for engineering of a variety of excited states of BEC containing vortices. In the ideal case the method allows one to generate genuine vortices with integer angular momenta. In the presence of imperfections in the absorption plate, typically more complex vortex patterns are generated.
We suggest an interference-based method of vortex detection, in analogy with methods used in nonlinear optics [15]. In the context of matter waves, similar methods were proposed in Refs. [16,17]. Our idea is to combine this method with the recently developed techniques of Bragg diffraction for BEC manipulation [18].

Before we turn to details, we should stress that the dynamical generation of vortices differs from the case of rotating traps, in which a pure vortex state with angular momentum $L_{z}=1$ (in units of $\hbar$ ) is selected in the process of reaching the equilibrium. In our case, generation of pure vortices requires the fine tuning of parameters that can hardly be achieved in experiments. Our method is suitable for the creation of generic states with vorticity [16], i.e., states with several vortex lines, around which the circulation of superfluid velocity does not vanish [19].

The dynamics of BECs, and thus the process of creation and evolution of vorticity at zero temperature are well described by the time-dependent Gross-Pitaevskii equation for the wave function $\psi(\mathbf{r}, t)$ :

$$
i \hbar \partial_{t} \psi=\left(\frac{-\hbar^{2} \nabla^{2}}{2 M}+V_{N L}(\mathbf{r}, t)+V_{t}(\mathbf{r})+V_{l}(\mathbf{r}, t)\right) \psi,
$$

where $V_{t}(\mathbf{r})=M\left(\omega_{x}^{2} x^{2}+\omega_{y}^{2} y^{2}+\omega_{z}^{2} z^{2}\right) / 2$ is the external trap potential, which we assume to be harmonic, $M$ is the mass of the atom, and $\omega_{x}, \omega_{y}, \omega_{z}$ are the trap frequencies. The nonlinear term $V_{N L}(\mathbf{r}, t)=g|\psi(\mathbf{r}, t)|^{2}$ describes the mean-field two-body repulsive interaction whose strength $g$ is related to the scattering length $a$ by $g=4 \pi N \hbar^{2} a / M$, where $N$ is the total number of condensated particles. The term $V_{l}(\mathbf{r}, t)$ describes an effective potential created by an external laser beam impinging on the condensate after passing through a plate with appropriately modulated absorption coefficient. In the following we will study the two-dimensional (2D) version of Eq. (1), in which we replace $g$ by $g / D$, where $D$ is the characteristic depth chosen such that the ground-state chemical potentials of the $3 \mathrm{D}$ and $2 \mathrm{D}$ systems are equal. 


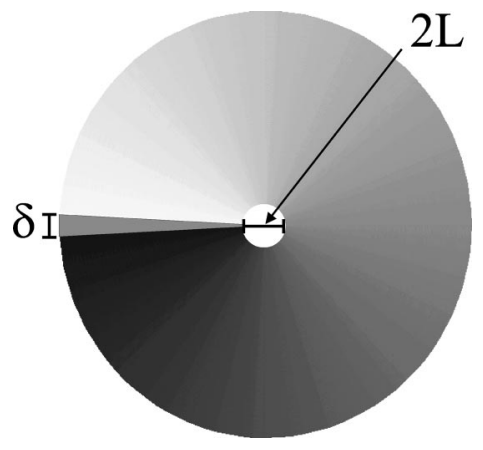

FIG. 1. Absorption plate. $L$ is the radius on which the absorption profile was smoothed in our simulations, whereas $\delta$ is the angular extension of the smoothing.

In this Rapid Communication we show that a short pulse of the light beam with a typical duration of the order of fractions of microseconds with properly modulated intensity profile creates vorticity in the Bose-Einstein condensate initially in its ground state. If the incident light is detuned far from the atomic transition frequency its main effect on atoms is to induce the Stark shift of internal energy levels. As the intensity of light depends on the position, the Stark shift will also be position dependent, as will the phase of the condensate.

The main feature characterizing a vortex is related to the particular behavior of the phase of the wave function at the vortex line: the phase "winds up" around this line, i.e., it changes by an integer multiple $m$ of $2 \pi$ on the path surrounding the vortex. Index $m$ is the winding number or the vortex charge. The light beam, before impinging on the atomic system, is shaped by an absorption plate whose absorption coefficient varies linearly with rotation angle $\varphi$ around the plate axis (see Fig. 1). As a result, $V_{l}(\mathbf{r}, t)$ depends on the distance from the propagation axis $\rho$, and the azimuthal angle $\varphi$.

In the ideal case, that absorption plate causes a real jump of the potential at, say, $\varphi=0$. In this case we can model the potential as being constant for $0 \leqslant t \leqslant T$ :

$$
V_{l}(\rho, \varphi)= \begin{cases}\hbar I \varphi \sin ^{2}(\pi \rho / 2 L) & \text { for } \rho \leqslant L, \\ \hbar I \varphi & \text { for } \rho>L\end{cases}
$$

and vanishing for other times. Here $T$ is the (square) pulse duration, whereas $I$ denotes the characteristic Stark shift (proportional to the far-off-resonant laser intensity); $L$ is the characteristic length scale, on which the absorption profile is smoothed in our calculations in the vicinity of the propagation-rotation axis. The use of more realistic temporal pulse shapes does not change the results significantly. In the following we will use square pulses, since it allows us to control the pulse area in a simpler manner.

The characteristic time and length scales for the BEC that we consider are milliseconds, and micrometers, respectively [20]. Note that in the regime of parameters when $T$ is short $(\simeq \mu \mathrm{s})$, and $\hbar I$ much larger than other energy scales, the dynamical effect of $V_{l}(\rho, \varphi)$ corresponds approximately to a "phase imprinting." Since during the interaction with the laser, all other terms in Eq. (1) can be neglected, the wave function after switching off the pulse becomes

$$
\psi(\mathbf{r}, T)=\exp \left[-i T V_{l}(\rho, \varphi) / \hbar\right] \psi(\mathbf{r}, 0)
$$

For the ideal case, choosing $I T=m$, we get the desired phase dependence characteristic for a pure vortex state. Unfortunately, the above picture is oversimplified, because the wave function (3) does not necessarily vanish at the vortex line $(\rho=0)$, which signifies infinite kinetic energy and its unphysical character. One has to solve the full dynamics of the system for $0 \leqslant t \leqslant T$, especially taking into account the kinetic-energy term. Moreover, realistic absorption plates cannot generate the singularity in the $\varphi$ dependence of $V_{l}$. For this reason we smooth the $\varphi$ dependence of the intensity $I$ on the scale of $\delta$ radians (see Fig. 1). "Phase imprinting", with this smoothed intensity distribution cannot generate pure vortex states-it can, however, as we shall see below, generate in a controlled way states with predetermined vorticity.

The Gross-Pitaevskii equation was solved using the split operator method in 2D ( $x$ and $y$ plane). The simulations were divided into two stages: the initial excitation stage of duration $T \simeq 0.16 \mu \mathrm{s}$, with about 1000 time steps, and the second stage with a characteristic time scale of $\mathrm{ms}$, and about $1000 \mathrm{steps} / \mathrm{ms}$. We have assumed that initially a condensate containing $N=100000$ rubidium atoms (with $a=5.8 \mathrm{~nm}$ ) was trapped in a disk-shaped trap of frequencies $\omega_{x}=\omega_{y}$ $=2 \pi \times 30 \mathrm{~Hz}, \omega_{z}=2 \pi \times 300 \mathrm{~Hz}$. In this case the condensate radius was about $15 \mu \mathrm{m}$. We have used $I=6$ $\times 10^{6} \mathrm{~Hz}$, and adjusted $T$ to obtain the desired values of the pulse area $(I T \simeq 1,2, \ldots)$. The $\rho$ dependence of the potential $V_{l}$ was smoothed with $L \simeq 1.7 \mu \mathrm{m}$, and the $\varphi$ dependence with $\delta \simeq 0.15$ radians. The smallest possible value of $\delta$ $\simeq 0.04$ radians was determined by the spatial grid size, which typically was $512 \times 512$ points in a $40 \mu \mathrm{m} \times 40 \mu \mathrm{m}$ box. Additionally, we studied the case when the laser was focused slightly off the trap symmetry axis. During the dynamics, after switching off the laser, the following quantities are conserved: wave-function norm, mean energy, and mean $z$ component of the angular momentum. They were monitored in order to control the accuracy of the numerics. The first two quantities were constant within the accuracy of the method; the third one in some cases exhibited slow variations of the order of a percent.

One of the most important problems concerning the investigation of vortices is finding an efficient method for their detection. Experimentally monitoring density profiles with the necessary resolution is difficult, since the vortex core is very small and hardly visible. The best way is to monitor the phase of the wave function in an interference measurement. Such interference measurements are routinely done in nonlinear optics [15]. In the context of matter waves, they were first proposed in Ref. [16] where the interference of two condensates moving towards one another was considered. If both condensates are in the ground state (no vortices), one expects the same interference fringes as those observed by Andrews et al. [21]. In the case of the interference of one condensate in the ground state with a second one in an $m$ $=1$ vortex state, a forklike dislocation in the interference pattern appears. The distance between the interference 
fringes is determined by the relative velocity of the condensates, which can be controlled experimentally. This is a very efficient and clear method of vorticity detection. It requires, however, the use of the two independent condensates.

We propose here to combine the interference method with the recently developed Bragg diffraction technique [18], which has been successfully used in the four-wave-mixing experiment [22]. The idea is to transfer part of the atoms coherently to another momentum state using one, or several, stimulated two-photon Raman scattering processes. The procedure is the following. (i) First we create the vortex, or vorticity state in the trap. (ii) We open the trap and let the condensate expand. (iii) When the density is reduced to values for which nonlinear interactions are negligible, we apply the Bragg pulses. Part of the wave function attains a phase factor, which signals the fact that the corresponding momentum was transferred to a part of the atomic sample [23]. The resulting wave function is the superposition of two vortex (or vorticity) states moving apart from each other, with a velocity that can be easily controlled by the choice of angle between the Bragg beams. In the following we will use velocities of the order of $1 \mathrm{~mm} / \mathrm{s}$, which allow for efficient detection after $4-5 \mathrm{~ms}$ when the vortices are about $5 \mu \mathrm{m}$ apart. (iv) Detection consists of optical imaging that is accomplished within a few micrometers. The interference patterns have a characteristic length scale of a few $\mu \mathrm{m}$.

We shall first discuss our results for the ideal case: $\delta$ $\leqslant 0.04$ radians, $I T=1$, and the laser focused exactly along the trap symmetry axis. In this case during the first stage of the dynamics angular momentum is transferred to the system. The mean of $L_{z}$ grows monotonically from 0 to about 1 (in units of $\hbar$ ), and stays close to 1 thereafter. The dispersion $\delta L_{z}$ grows within the pulse duration to about 5 , and returns to about 0 for $t=T$. In the second stage of the dynamics, a hole in the density profile appears, and the density acquires a stationary state within a fraction of a millisecond. This time scale corresponds to the inverse of the characteristic frequency, determined by the mean-field energy in the condensate, $g / \hbar V$, where $V$ is the volume of the condensate ( $V$ $\simeq \pi 15 \times 15 \times 4 \mu \mathrm{m}^{3}$ ). It is the time required by the second sound to travel over a distance of the order of the healing length. The density distribution thereafter remains constant on the scale of 10-20 ms. The dispersion of the angular momentum, $\delta L_{z}$, exhibits a stepwise growth to about $\delta L_{z} \simeq 5$ with fast growth periods of about $0.5 \mathrm{~ms}$ duration, followed by slow growth stages of a few milliseconds duration. We observed a slight movement of the vortex off the center of the trap.

In Fig. 2(a) we present typical simulation results obtained a few milliseconds after the creation of a vortex using the detection method of [16] based on the interference of a condensate in the vortex state with a condensate in the planewave state. The characteristic forklike pattern reflects the fact that the phase winds up by $2 \pi$, as one circulates around the vortex line. Figure 2(b) shows the simulation of the interference pattern for the method of detection proposed by us. A few milliseconds after applying the Bragg pulses we can clearly see a double fork structure, as one should expect for this case. The forks are oriented in opposite directions, because the condensates have the same helicity but opposite velocities.
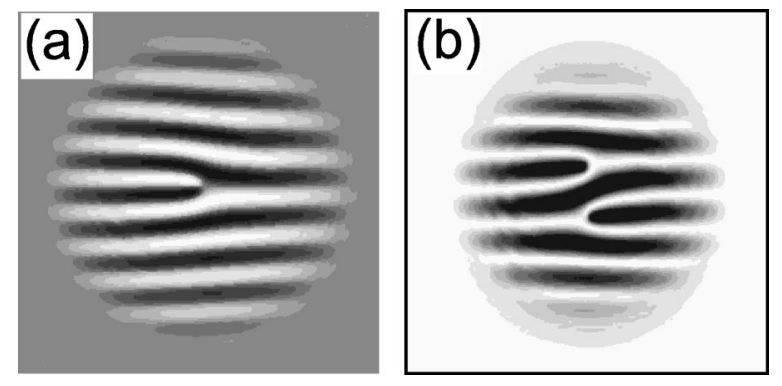

FIG. 2. Interference pattern of the vortex state $(5 \mathrm{~ms}$ after phase imprinting): (a) superimposed with a plane wave of $k=1.8 \mu \mathrm{m}^{-1}$ moving "upward" in the plane of the figure; (b) $5 \mathrm{~ms}$ after applying Bragg pulses transferring momentum to a part of the condensate (along the vertical axis in the plane of the figure). The ideal case of the equal splitting of the whole condensate has been assumed. The incident pulse was focused at the trap center and had a sharp step in the intensity profile $(\delta=0.04)$.

It is interesting to compare this ideal result with more realistic simulations obtained for $\delta=0.15$, keeping the laser focused at the center of the trap and the pulse area $I T=1$. In this case one excites a vorticity state that is not pure. The mean $L_{z}$ after the laser pulse is close to zero, as it should be in the absence of the phase discontinuity. The dispersion $\delta L_{z}$ behaves similarly as before, although it grows a little faster. The density profile now exhibits the hole in the center but also a number of deep minima, or holes at $\varphi=0$ in the outer regions of the condensate. While the density distribution is difficult to interpret, the interference method allows us to interpret them very clearly. Application of the plane-wave method indicates two vortices of opposite winding number (topological charge) and perhaps two more close to the edge of the condensate [Fig. 3(a)]. This basic structure is doubled, but nevertheless is clearly recognizable using interference combined with Bragg scattering [Fig. 3(b)].

Finally, in Fig. 4 we present numerical results that mimic other possible experimental imperfections: the offset of the laser focus by $1.7 \mu \mathrm{m}$ off the center of the trap (in the positive direction of the $x$ axis), combined with the smoothed $\varphi$ dependence of the absorption profile $(\delta=0.15)$. In this case the behavior of $L_{z}$ and $\delta L_{z}$ is similar to the case presented in Fig. 3. The interference patterns, obviously, are more noisy and irregular. Still one can clearly recognize forklike dislocations corresponding to vortices using the
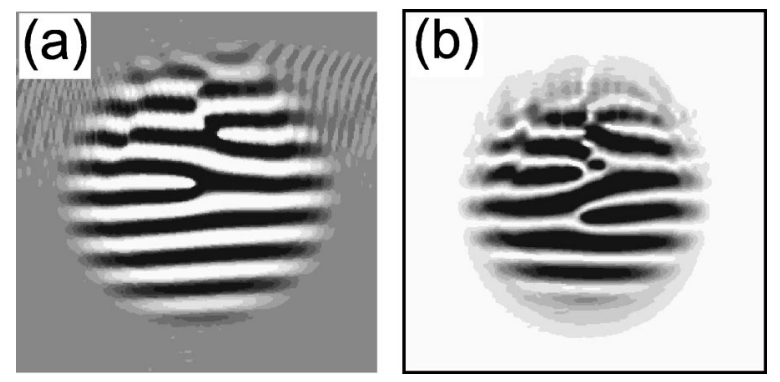

FIG. 3. Interference pattern of the vorticity state $(6 \mathrm{~ms}$ after the phase imprinting): (a) after superimposing with a plane wave as in Fig. 1(a); (b) after applying the Bragg pulses as in Fig. 1(b). The incident pulse was focused in the trap center and its intensity profile was smoothed over an angle $\delta=0.15$. 

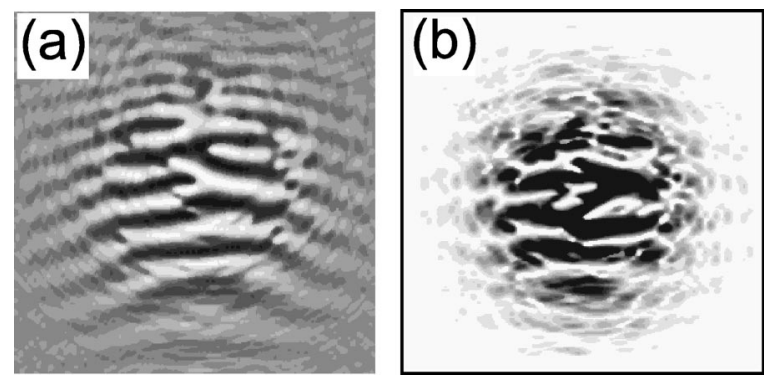

FIG. 4. Interference pattern of the vorticity state $(8.5 \mathrm{~ms}$ after phase imprinting): (a) after superimposing with a plane wave as in Fig. 1(a); (b) after applying the Bragg pulses as in Fig. 1(b). The incident pulse was focused $1.7 \mu \mathrm{m}$ off the trap center and its intensity profile was smoothed over an angle $\delta=0.15$.

plane-wave interference method [Fig. 4(a)] and the method proposed here [Fig. 4(b)].

In our numerical simulations we have also studied situations in which the area of the phase imprinting pulse is large,
$I T>1$. In these cases typically a number of singly charged vortices aligned in geometric patterns similar to those obtained in rotating traps [7] are created.

Summarizing, we have proposed a method of vortex generation in trapped Bose-Einstein condensates. The method employs optical potentials induced by passing a laser beam through an absorption plate with an absorption coefficient that depends on the azimuthal angle $\varphi$. We have also proposed a method of detecting vortices and vorticity by combining the known interference method with the recently developed Bragg scattering techniques to coherently transfer atoms into a selected momentum state.

Note added in proof. Recently the vortices in two component condensates have been obtained experimentally [24].

We acknowledge fruitful discussions with $\mathrm{K}$. Bongs, $\mathrm{S}$. Burger, M. Brewczyk, J.I. Cirac, S. Dettmer, W. Królikowski, and K. Rzążewski. This work is supported by the SFB 407 of the Deutsche Forschungsgemeinschaft. M.G. is partially supported by the KBN, Grant No. 2 P03B 13015.
[1] M. J. Anderson, J. R. Ensher, M. R. Matthews, C. E. Wieman, and E. A. Cornell, Science 269, 198 (1995).

[2] K. B. Davis, M. -O. Mewes, M. R. Andrews, N. J. van Druten, D. S. Durfee, D. M. Kurn, and W. Ketterle, Phys. Rev. Lett. 75, 3969 (1995).

[3] C. C. Bradley, C. A. Sackett, J. J. Tollett, and R. G. Hulet, Phys. Rev. Lett. 75, 1687 (1995); C. C. Bradley et al., ibid. 78, 985 (1997); D. G. Fried et al., Phys. Rev. Lett. 81, 3807 (1998).

[4] W. Ketterle and E. A. Cornell (private communication); W. Phillips and K. Helmerson (private communication).

[5] R. J. Marshall et al., Phys. Rev. A 59, 2085 (1999); P. D. Drummond and J. F. Cornay, e-print cond-mat/9806315.

[6] F. Dalfovo and S. Stringari, Phys. Rev. A 53, 2477 (1996).

[7] D. A. Butts and D. S. Rokhsar, Nature (London) 397, 327 (1999).

[8] D. L. Feder, Ch. W. Clark, and B. I. Schneider, Phys. Rev. Lett. 82, 4956 (1999).

[9] B. Jackson, J. F. McCann, and C. S. Adams, Phys. Rev. Lett. 80, 3903 (1998).

[10] B. M. Caradoc-Davies, R. J. Ballagh, and K. Burnett, e-print cond-mat/9902092.

[11] K.-P. Marzlin and W. Zhang, Phys. Rev. A 57, 3801 (1998); 57, 4761 (1999).

[12] R. Dum et al., Phys. Rev. Lett. 80, 2972 (1998).

[13] K.-P. Marzlin, W. Zhang, and E. M. Wright, Phys. Rev. Lett. 79, 4728 (1997).

[14] The stability of vortices in nonrotating traps of various shapes has been very intensively studied recently: D. S. Rokhsar, Phys. Rev. Lett. 79, 2164 (1997); e-print cond-mat/9709212; A. A. Svidzinsky and A. L. Fetter, Phys. Rev. A 58, 3168 (1998); H. Pu et al., ibid. 59, 1533 (1999); T. Isoschima and
K. Machida, ibid. 59, 2203 (1999); Th. Busch and J. Anglin, e-print cond-mat/9811147; e-print cond-mat/9809408; J. J. Garcia-Ripoll et al., e-print quant-ph/9812014; P. O. Fedichev, A. E. Muryshev, and G. V. Shlyapnikov, e-print cond-mat/9905062; P. O. Fedichev and G. V. Shlyapnikov, e-print cond-mat/9902204; A. E. Muryshev et al., e-print cond-mat/9811408.

[15] K. Staliunas, C. O. Weiss, and G. Slekys, Horizons of World Physics, edited by M. Vasnetsov and K. Staliunas (Nova Science Publishers, Commack, NY, 1999), Vol. 228; I. V. Basistiy et al., Opt. Commun. 103, 422 (1993); L. V. Kreminskaya, M. S. Soskin, and A. I. Khizhnyak, ibid. 145, 377 (1998); J. F. Nye and M. V. Berry, Proc. R. Soc. London, Ser. A 336, 165 (1974).

[16] E. L. Bolda and D. F. Walls, Phys. Rev. Lett. 81, 5477 (1998); J. Tempere and J. T. Devreese, Solid State Commun. 108, 993 (1998).

[17] F. Dalfovo et al., Rev. Mod. Phys. 71, 463 (1999).

[18] M. Kozuma et al., Phys. Rev. Lett. 82, 871 (1999).

[19] D. Pines and Ph. Nozières, Theory of Quantum Liquids (Benjamin, Reading, MA, 1966).

[20] K. Bongs et al. (unpublished).

[21] M. R. Andrews et al., Science 275, 637 (1997); M.-O. Mewes et al., Phys. Rev. Lett. 78, 582 (1997).

[22] L. Deng et al., Nature (London) 398, 218 (1999).

[23] If the resonant width of the Raman transition is narrower than the wave-function spreading in the momentum space, not all momentum components can experience the shift. In that case the contrast of interference fringes will be reduced. One can, however, correct for this by adiabatically opening the trap before applying Bragg pulses.

[24] M. R. Matthews et al., e-print cond-mat/9908209. 